\title{
Die neue Satzung der DIVI
}

A ufbruchstimmung bei der Deutschen Interdisziplinären Vereinigung für Intensiv- und Notfallmedizin (DIVI): Nach langem Ringen hat die Mitgliederversammlung der DIVI am 10. Juni 2008 eine neue Satzung verabschiedet. Damit legten die Mitglieder den Grundstein für eine inhaltliche und strukturelle Weiterentwicklung der DIVI. Die Intensiv- und Notfallmedizin in Deutschland wird so entscheidend gestärkt.

Ab sofort vertritt die DIVI nicht nur die wissenschaftlichen Fachgesellschaften und Berufsverbände aller Fachgebiete, die Intensiv- und Notfallmedizin betreiben. Sie vertritt auch Ärztinnen und Ärzte als Einzelmitglieder und öffnet sich zudem nicht ärztlichen Personen.

Die neue DIVI gliedert sich in 3 Einheiten. Die Division A ist die Einheit der wissenschaftlichen Fachgesellschaften und Berufsverbände. Die Mitglieder sind nach der jeweils gültigen Weiterbildungsordnung zu einer Zusatzweiterbildung Intensivmedizin bzw. Notfallmedizin berechtigt (DIVI-FB). Ferner gibt es 2 Divisionen für Einzelmitglieder. Die Division B (DIVI-MG-Ärzte) steht den Ärzten offen: Fachärzten mit der Zusatzweiterbildung Intensivmedizin bzw. Notfallmedizin (ordentliche Mitglieder) und Ärzten in der Weiterbildung (außerordentliche Mitglieder). In der Division C sind nicht ärztliche Mitglieder angesiedelt, die in Intensivund Notfallmedizin tätig sind (DIVI-MG-Nichtärzte).

Die Divisionen A und B setzen sich zusammen aus den Fachgebieten Anästhesiologie, Chirurgie, Innere Medizin, Kinder- und Jugendmedizin sowie Neurologie und Neurochirurgie. Die 5 Fachgebiete und die Divisionen DIVI-FB und DIVI-MG-Ärzte sind im Präsidium gleichberechtigt vertreten.

Aus vereinsrechtlichen Gründen war es notwendig, zunächst ein Interimspräsidium zu bilden.
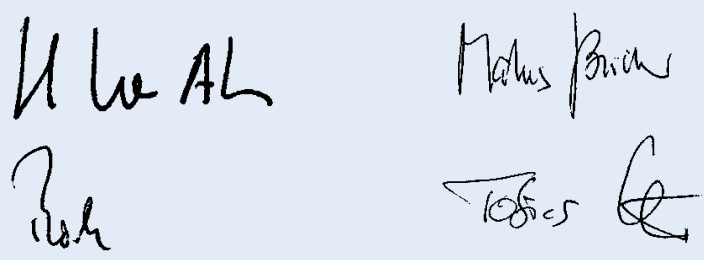

Es setzt sich wie folgt zusammen:

- Präsident: Prof. Dr. A. Markewitz (Chirurgie)

- Vizepräsidenten: Prof. Dr. Dr. H. Van Aken (Anästhesiologie), Prof. Dr. G. Sybrecht (Innere Medizin)

- Schatzmeister: Prof. Dr. D. Schneider (Neurochirurgie/Neurologie)

- Generalsekretär/Schriftführer: Prof. Dr. G. Jorch (Kinder- und Jugendmedizin)

Weitere Präsidiumsmitglieder sind Prof. Dr. E. Muhl (Chirurgie), Prof. Dr. M. Quintel (Anästhesiologie), Prof. Dr. H. Stopfkuchen (Kinder- und Jugendmedizin) Prof. Dr. E. Rickels (Neurochirurgie/Neurologie) und Prof. Dr. T. Welte (Innere Medizin).

Die neue Satzung muss jetzt noch vom Amtsgericht Düsseldorf in das Vereinsregister eingetragen werden. Eine bürokratischer Akt, von dem man im Voraus nicht sagen kann, welche Hindernisse sich auftun. Wir hoffen, dass dieser Vorgang schnell erledigt sein wird, so dass das Interim Präsidium am 3.12. zurücktreten kann und am gleichen Tag die Neuwahl in der Mitgliederversammlung um 17.15 Uhr auf dem DIVIKongress in Hamburg stattfinden kann.

Den endgültigen Text der Satzung finden Sie unter „Infoservice/Aktuelles“ auf folgenden Webseiten: www.dgai.de und www.bda.de.

Die Herausgeber der Zeitschrift Intensivmedizin up2date ermutigen die Leser der Zeitschrift, Mitglied der DIVI zu werden. Personen, die Mitglied einer wissenschaftlichen Gesellschaft oder eines Berufsverbands sind, der der DIVI angehört, erhalten einen deutlichen Beitragsrabatt.

Mit herzlichen Grüßen

Ihre Herausgeber

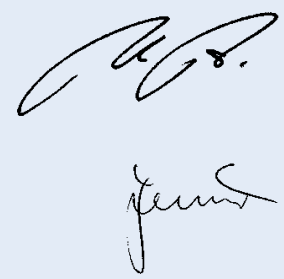

